\title{
CONDITIONS FOR EQUALITY OF THE MACKEY AND STRICT TOPOLOGIES
}

\author{
BY F. DENNIS SENTILLES
}

Communicated by R. C. Buck, August 4, 1969

1. Introduction. In $1958 \mathrm{R}$. C. Buck [2] made a study of the $\beta$ or strict topology (introduced in [1] and named for its resemblance to a topology of Beurling) on the space $C(S)$ of bounded continuous functions on a locally compact space $S$ under which the dual of $C(S)_{\beta}$ is the space $M(S)$ of bounded regular Borel measures on $S$. In 1966, J. B. Conway [4], [5] showed that when $S$ is $\sigma$-compact (or even paracompact) the strict topology is the Mackey topology on $C(S)_{\beta}$ - the finest locally convex topology on $C(S)$ for which the dual is $M(S)$. However, when $S$ is the space of ordinals $[1, \Omega)$ less than the first uncountable ordinal $\Omega$, Conway showed that $\beta$ is not the Mackey topology on $C(S)_{\beta}$.

In [10] Wang studied the strict topology generalized to Banach algebras. More recently, the author and D. C. Taylor [8] studied the strict topology defined by a Banach algebra $B$ with approximate identity on a left Banach $B$-module $X$ by way of the seminorms $x \rightarrow\|T x\|$, one for each $T \in B$ such that $B$ separates points of $X$. No necessary or sufficient conditions were obtained for which this general strict topology $\beta$ is the Mackey topology on $X_{\beta}$. In this paper, sufficient conditions are given in order that $\beta$ be the Mackey topology on $X_{\beta}$, with our aim being to obtain conditions which in some sense differentiate between the case where $S$ is $\sigma$-compact, as opposed to $S=[1, \Omega)$, but in the general setting of $[8]$. A crucial step in the argument is provided by some results which generalize that of Dorroh [6] and show that the continuity of linear maps on $X_{\beta}$ is of ten determined by their continuity on norm bounded sets in $X$.

2. Some observations. It is a known result that if a space $E$ has its Mackey topology $\tau=\tau\left(E, E^{\prime}\right)$, then every continuous map on $E$ into a locally convex space $F$ with its weak topology is continuous on $E$ into $F$ with its given topology. Actually one can prove

AMS Subject Classifications. Primary 4601, 4665; Secondary 4650.

Key Words and Phrases. Locally convex spaces, strict topology, bounded strict topology, Mackey topology, Banach modules, operator algebras. 
THEOREM 2.1. E has the topology $\tau$ iff every continuous linear map on $E$ into a space $C(T)$ with the topology of pointwise convergence on $T$ is continuous with the supremum norm topology on $C(T)$, for every compact Hausdorff space $T$.

This is not a very deep result, but it did motivate our approach to the problem. In $\$ 4$ we consider the continuity of linear maps on $X_{\beta}$.

In [8], (where we assume $B$ separates points of $X$ and from which all further notation is taken) it is shown that $\beta$ is the given norm topology on $X$ iff $B$ contains a one-to-one operator on $X$ with closed range. Our approach is to weaken this latter condition and obtain $\beta=\tau$. We note that in the case $B=C_{0}(S), X=C(S)$, there is a $\phi$ $\in C_{0}(S)$ such that $f \rightarrow \phi \cdot f$ is one-to-one for $f \in C(S)$, when $S$ is $\sigma$-compact, but not when $S=[1, \Omega)$.

However, we also note that when $X$ is a separable Hilbert space and $B$ is the algebra of compact operators in $X$ with countable approximate unit consisting of projections onto finite dimensional subspaces, then, as our Theorem 3.3 asserts, there is a one-to-one $A \in B$, but since $X$ is reflexive and $X=X_{\theta}=\{T x: T \in B, x \in X\}$, the Mackey topology on $X_{\beta}$ is the norm topology. Hence a further condition is needed to obtain $\beta=\tau$. Our Theorem 5.1 provides such a condition.

3. One-to-one operators in $B$. Suppose that $\left\{E_{\lambda}\right\}$ is a two-sided approximate identity for $B$ bounded by 1 and that there is an $A \in B$ such that $A$ is one-to-one on $X$ and such that for each $\lambda, E_{\lambda} A^{-1}$ is a bounded linear operator on $A(X) \subset X_{\boldsymbol{e}}$. It follows that one can obtain a sequence $\left\{\lambda_{n}\right\}$ such that

3 (a) $\lambda_{n+1} \geqq \lambda_{n}$ and $\left\|E_{\lambda_{n}} E_{\lambda_{k}}-E_{\lambda_{k}}\right\| \leqq 1 / 2^{n}$ for $k \leqq n-1$

3(b) If $\beta \geqq \lambda_{n}$ then each of $\left\|E_{\lambda} A-A\right\|,\left\|A E_{\lambda}-A\right\|$ and $\left\|A E_{\lambda}-E_{\lambda} A\right\|$ $\leqq 1 / 2^{n}$.

Upon letting $E_{n}=E_{\lambda_{n}}$ we have

TheOREM 3.1. (a) For $x \in X, E_{n} x \rightarrow x$ in the strict topology.

(b) If $A(X)$ is dense in $X_{e}$, then $E_{n} x \rightarrow x$ in the norm topology for $X_{e}$ for all $x \in X_{\text {e }}$.

Letting $\omega$ denote the topology on $X$ induced by the norm $x \rightarrow\|A x\|$ we have

THEOREM 3.2. (a) $\kappa \leqq \omega \leqq \beta$.

(b) $\kappa=\omega=\beta$ on $\sigma^{\prime}$-bounded sets.

(c) If $X$ is $\omega$-complete, then $\beta$ is the given norm topology on $X$.

While in 3.1, $\left\{E_{n}\right\}$ need not be an approximate identity for $B$ we can obtain the partial converse. 
TheOREM 3.3. Suppose $B$ contains a bounded sequence $\left\{G_{n}\right\}$ such that $G_{n} x \rightarrow x$ in the strict topology for each $x \in X$. Then, if $1 \leqq a_{n} \rightarrow \infty$, then there is a 1-1 $A \in B$ such that $\left\|G_{n} A^{-1}\right\| \leqq a_{n}$ on $A(X)$.

The proof of Theorem 3.3 depends on showing that

$$
H=\bigcup_{n=1}^{\infty}\left(1 / a_{n}\right) G_{n}^{\prime}\left(U^{0}\right)
$$

is equicontinuous in $X_{\beta}^{\prime}$, where $U^{0}$ is the polar of the unit ball $U$ in $X_{e}$.

We note that one can have $\beta=\tau$ and not have a 1-1 operator $A$ in $B$, for when $S$ is not $\sigma$-compact and $C(S)_{\beta}$ is Mackey (e.g., if $S$ is paracompact) no $\phi \in C_{0}(S)$ can separate points of $C(S)$ since any such $\phi$ has $\sigma$-compact support. However, it is important to note that when $S$ is paracompact, then $S$ is the union of mutually disjoint open and closed $\sigma$-compact spaces and it is this fact which allows one to show that $\beta$ is the Mackey topology on $C(S)_{\beta}$.

Finally we note,

Theorem 3.5. If $A$ and $\left\{E_{\lambda}\right\}$ are such that $\sup _{\lambda}\left\|E_{\lambda} A^{-1}\right\|<\infty$ on $A(X)$, then $\beta$ is the given norm topology on $X$ whenever the $\beta$ bounded sets are norm bounded.

4. Continuity of linear maps on $X_{B}$. Our Theorem 3.2 indicates that $\beta$ can be a relatively nice topology on bounded sets. In [5] Dorroh showed that the "bounded strict topology" on $C(S)$, whose base of neighborhoods consisted of absolutely convex absorbent sets $W$ such that $W \cap B_{r} \supset B_{r} \cap V$ for some $\beta$ neighborhood $V$, for each ball $B_{r}$ of radius $r>0$, is equivalent to $\beta$. We extend this result to the general case of the strict topology studied in [8]. For a general study of topologies of this type see [3].

Theorem 4.1. If $B_{r}=\{x \in X:\|x\| \leqq r\}$ and if $W$ is a $\beta$-closed, absolutely convex, absorbent set in $X$ such that for each $r>0$ there is a $\beta$ neighborhood $V$ of 0 such that $W \cap B_{r} \supset B_{r} \cap V$, then $W$ is a $\beta$ neighborhood of 0 .

CoROLlary 4.2. (a) $\beta$ is the finest locally convex topology on $X$ agreeing with $\beta$ on each set $B_{r}$ iff every linear functional on $X$ which is $\beta$ continuous on each $B_{r}$ is $\beta$ continuous on $X$.

(b) When $X_{\beta}^{\prime}=X_{e}^{\prime}$ the equivalent conditions in (a) hold.

Corollary 4.3. (a) $A$ weakly continuous linear operator $L$ on $X_{\beta}$ into a locally convex space $E$ is continuous iff $L$ is continuous at 0 on each set $B_{r}$. 
(b) If $X_{\beta}^{\prime}=X_{e}^{\prime}$, then a linear mapping $L: X_{\beta} \rightarrow E$ is continuous iff $L$ is continuous at 0 on each set $B_{r}$.

Since $\beta=\omega=\kappa$ on each set $B_{r}$ it follows that when an operator $A$ of the type considered in $\$ 3$ exists, then $A$ determines the continuity of linear maps on $X_{\beta}$ when $X_{\beta}^{\prime}=X_{e}^{\prime}$.

5. The main theorem. We suppose $B$ contains an operator $A$ with the properties assumed at the start of $\$ 3$ and that $\left\{E_{n}\right\}$ has been chosen to have properties $3(\mathrm{a})$ and $3(\mathrm{~b})$.

ThEOREM 5.1. If $X_{\beta}$ is complete, then $\beta=\tau$ when the following condition holds: If $\left\{x_{k}\right\} \subset X_{e} \cap B_{r}$ is a sequence such that

(a) $\left\|A x_{k}\right\| \leqq 1 / 2^{k-1}$ and

(b) $\left\|E_{n_{k}} x_{j}\right\| \leqq 1 / 2^{j-1}$ for $k \leqq j-1, j=1,2, \cdots$ for a subsequence $\left\{E_{n_{k}}\right\} \subset\left\{E_{n}\right\}$, then

(c) $\sup ^{P}\left\|\sum_{k=1}^{P} E_{n_{k}} x_{k}\right\|<\infty$.

To sketch the proof of Theorem 5.1, suppose $H$ is a $w^{*}$ compact absolutely convex set in $X_{\beta}^{\prime}$ such that the polar $H^{0}$ of $H$ in $X$ is not a $\beta$-neighborhood of 0 . Then by Theorem 4.1 and [8, Theorem 3.1] there is an $r>0$ such that $H^{0} \cap B_{r} \supset B_{r} \cap V_{T}$ holds for no $T \in B$, where $V_{T}=\{x \in X:\|T x\| \leqq 1\}$.

Let $c_{n}=\max \left\{\left\|E_{k} A^{-1}\right\|: 1 \leqq k \leqq n\right\}$. It then follows that there is an $x_{1} \in V_{A} \cap B_{r} \cap X_{e}$ and an $x_{1}^{\prime} \in H$ and $E_{n_{1}} \in\left\{E_{k}\right\}$ such that $\left|\left\langle E_{n_{1}} x_{1}, x_{1}^{\prime}\right\rangle\right|$ $\geqq 1$. We then obtain $x_{2} \in(1 / 2) c_{n_{1}} V_{A} \cap B_{r} \cap X_{e}, E_{n_{2}}, x_{2}^{\prime} \in H$ such that $\left|\left\langle E_{n_{2}} x_{2}, \quad x_{2}^{\prime}\right\rangle\right| \geqq 1$ and note that $\left\|E_{n_{1}} x_{2}\right\|=\left\|E_{n_{1}} A^{-1} A x_{2}\right\| \leqq c_{n_{1}}\left\|A x_{2}\right\|$ $\leqq 1 / 2$.

Continuing this way we obtain sequences, $\left\{x_{i}\right\} \in B_{r},\left\{E_{n_{i}}\right\} \subset\left\{E_{k}\right\}$, $\left\{x_{i}^{\prime}\right\} \subset H$ such that $\left|\left\langle E_{n_{i}} x_{i}, x_{i}^{\prime}\right\rangle\right| \geqq 1$ and $\left\|E_{n_{j}} x_{i}\right\| \leqq 1 / 2^{i-1}$ for $j \leqq i-1$. From our hypothesis, if $\left\{a_{n}\right\} \in l_{\infty}$, then the sequence $y_{n}=\sum_{i=1}^{n} a_{i} E_{n_{i}} x_{i}$ is norm bounded in $X$ and $\omega$-cauchy by choice of $x_{i+1} \in\left(1 / 2^{i}\right) c_{n_{i}} V_{A}$. From 3.2(b), $L\left\{a_{i}\right\}=\lim y_{n}$ exists in $X_{\beta}$.

Since by our hypothesis and the uniform boundedness principle applied to $l_{\infty}, L$ maps bounded sets into bounded sets, then from 3.2(b) and 4.3(a) it follows that $L$ is continuous on $l_{\infty}$, with the strict topology defined by $c_{0}$, into $X_{\beta}$. Hence $L^{\prime}(H)$ is $\beta$-equicontinuous in $\left(l_{\infty}\right)_{\beta}^{\prime}=l_{1}$. But this is contradicted by the inequalities $\left|\left\langle E_{n_{i}} x_{i}, x_{i}^{\prime}\right\rangle\right| \geqq 1$ for $i=1,2, \cdots$.

The reader familiar with [4] or [5] will note some similarity of argument in the above proof. We note also that it is often more natural to view condition (c) in terms of the equivalent (by virtue of (b)) condition 


$$
\sup _{P}\left\|\sum_{k=1}^{P}\left(E_{n_{k+1}}-E_{n_{k}}\right) x_{k+1}\right\|<\infty \text {. }
$$

Finally, suppose that $X$ is a Banach algebra and $B$ is a closed commutative right ideal in $X$ which is a sup norm algebra with approximate identity $\left\{e_{\lambda}\right\}$ bounded by 1 . We then have

CoROLlary 5.2. If there is an $a \in B$ such that $a x=0$ implies $x=0$ for $x \in X$ and such that for each $\lambda$ there is a number $k_{\lambda}$ such that $\|a x\| \leqq 1$ implies $\left\|e_{\lambda} x\right\| \leqq k_{\lambda}$, then $\beta$ is the Mackey topology on the complete locally convex space $\operatorname{Hom}_{B}\left(B, X_{e}\right)_{\beta}$.

Regarding further applications, it is clear that our condition fails for the example mentioned at the end of $\$ 2$. In [8] several examples in which $\beta \neq \tau$ are given, notably the strict topology on $L^{P}(G)$, $1<P<\infty$, defined by $L^{1}(G)$. The most interesting case is that of the strict topology on $M(G)$ defined by $L^{1}(G)$ where it is not so clear that our condition fails, as it must for $G$ the group of real numbers because of the following example. If $e_{n}(x)=-n^{2}|x|+n$ for $|x| \leqq 1 / n$ and 0 for $|x|>1 / n$, then the collection of uniformly continuous functions $f_{n}(x)=(1 / n)\left[e_{n}(1 / n+x)+e_{n}(1 / n-x)\right]$ in $M(G)_{\beta}^{\prime}$ is weak ${ }^{*}$ compact but cannot be $\beta$-equicontinuous [8, Theorem 4.8], since $\left\{e_{n}\right\}$ is an approximate identity for $L^{1}(G)$ and $\left\|e_{n}^{*} f_{n}-f_{n}\right\|_{\infty} \geqq 1 / 4$ for all $n$. One would like to determine whether our condition in 5.1 is necessary for $\beta=\tau$.

Of the examples of the strict topology considered in [8], the only remaining one is that defined by the Banach algebra $B$ of compact operators in a Hilbert space $H$ on the left $B$-module $X$ of all bounded operators in $H$. Here Taylor [9] has shown that the Mackey topology on $X_{\beta}$ is not $\beta$, but (when $H$ is separable) is the "double-strict" topology obtained in the natural way by considering $X$ as a left and right $B$-module simultaneously and has extended this result to $B^{*}$-algebras with countable approximate identity.

\section{REFERENCES}

1. R. C. Buck, Operator algebras and dual spaces, Proc. Amer. Math. Soc. 3 (1952), 681-687. MR 14, 290.

2. - Bounded continuous functions on a locally compact space, Michigan Math. J. 5 (1958), 95-104. MR 21 \#4350.

3. H. S. Collins, Completeness and compactness in linear topological spaces, Trans. Amer. Math. Soc. 79 (1955), 256-280. MR 16, 1030.

4. J. B. Conway, The strict topology and compactness in the space of measures, Bull. Amer. Math. Soc. 72 (1966), 75-78. MR 32 \#4509. 
5. - The strict topology and compactness in the space of measures. II, Trans Amer. Math. Soc. 126 (1967), 474-486. MR 34 \#6503.

6. J. R. Dorroh, The localization of the strict topology via bounded sets, Proc. Amer. Math. Soc. 20 (1969), 413-414.

7. A. P. Robertson and W. J. Robertson, Topological vector spaces, Cambridge Tracts in Math. and Math. Phys., no. 53, Cambridge Univ. Press, New York, 1964. MR 28 \#5318.

8. F. D. Sentilles and D. C. Taylor, Factorizations in Banach algebras and the general strict topology, Trans. Amer. Math. Soc. 142 (1969), 142-152.

9. D. C. Taylor, The strict topology for double-centralizer algebras (to appears).

10. Ju-kwei Wang, Multipliers of commutative Banach algebras, Pacific J. Math. 11 (1967), 1131-1149.

University of Missouri, Columbia, Missouri 65201 